\title{
Theoretical Modeling and Implementation of Traveling Wave Sensor Based on PCB Coils
}

\author{
Zewen Li, Tuofu Deng, Xiangjun Zeng, Feng Deng, and Lei Shu \\ Hunan Province Key Laboratory of Smart Grids Operation and Control, School of Electrical and Information Engineering, \\ Changsha University of Science and Technology, Hunan 410076, China
}

Correspondence should be addressed to Zewen Li; lzw0917@163.com

Received 10 October 2014; Accepted 24 April 2015

Academic Editor: Aldo Minardo

Copyright (C) 2015 Zewen Li et al. This is an open access article distributed under the Creative Commons Attribution License, which permits unrestricted use, distribution, and reproduction in any medium, provided the original work is properly cited.

Based on analyzing characteristics of Rogowski coil, a new type of PCB traveling wave sensor with simple structure, high linearity, and anti-interference ability is proposed. The sensor has fine physical structure, which can effectively resist external electromagnetic interference by anti-interference measurement. In addition, it can greatly improve mutual inductance based on simple combinations. Simulations show that the new PCB traveling wave sensor can validly extract and deliver traveling wave signal and therefore realize fault location and protection accurately.

\section{Introduction}

With the power system expanding, the voltage class promoting, and higher network security requirements, accurate fault location becomes an important safeguard for fast faultclearing and improving of the power system [1-5]. The action time of travelling wave protection is much less than the lower frequency protection $[6,7]$. The accuracy of travelling wave signal detection directly influences the accuracy of travelling wave fault location and the reliability of travelling wave protection. So accurate detection of travelling wave signal has become the direction of travelling wave technology [8-11].

Current transformer and inductive voltage transformers can transfer the highest band signal and polarity of wave head without delaying $[12,13]$. It can be used for fault travelling wave protection and positioning analysis based on travelling wave head. But complicated software methods are necessary to identify and extract the wave head effectively. In Canada BC Hydro's $500 \mathrm{KV}$ transmission grid, fault travelling wave positioning system was installed. The sensor of travelling wave is a small electric reactor, in series with CVT's ground electrode to extract the voltage travelling wave signal $[14,15]$. The system can detect all kinds of travelling wave signals effectively. But because the primary system wiring need be changed when electric reactor is installed, this does not meet
China's power system operation standard so it is hard to be applied and spread. In [16, 17], a dedicated travelling wave sensor based on Rogowski coil theory was studied, muff-coupling on CVT's ground wire. Measure the current travelling wave at which ground through CVT can reflect the line voltage travelling wave. It has no direct electric connection with primary equipment and has advantages such as small delay errors, good frequency response, and high sensitivity. But the induction coil of travelling wave sensor need be wound manually or winding machine. It is not so well distributed on the secondary side winding. The coefficients of mutual induction and anti-interference are of big errors even producing the same time. Coil's transient response is hard to remain the same and with low stability. It is hard to meet the demand of nanosecond synchronization accuracy in traveling-wave detection.

In order to ensure the consistency for different coefficient of mutual inductance of traveling wave sensor, to boost antiinterference of coil, the primary conductor and secondary coil are both printed on the PCB board. Their locations were relatively stable and coiled uniformly. The consistency for different coefficient of mutual inductance of traveling wave sensor and the magnetic coupling are enhanced. A unique spiral winding method is adopted to wind the secondary coil to resist outside magnetic interference. 


\section{Operating Characteristics of Rogowski Coil}

Rogowski coil is a fairly mature current measuring device consisting of a bandpass filter with ideal amplitude-frequency, phase-frequency, and transient characteristics, which has been widely used to measure large current surge. Taking Rogowski coil to measure pulsed current, the measured signal is almost unrestricted. Rogowski coils are nonsaturated under any circumstances, thus being able to measure nanosecond rise time of current. It also has nondirect electrical contact with the primary side of the circuit. Clearly, the simple implementation of the first two requirements of the sensor and the detection delay is very short.

Rogowski coil has two kinds of working status: the status of self-integration and differentiation [18]. Rogowski coil analyzed in this paper is under the status of self-integration. In equivalent circuit 1 , there are equations as follows according to Kirchhoff's current law:

$$
\begin{aligned}
-M \frac{d i_{1}}{d t} & =L \frac{d i_{2}}{d t}+r i_{2}+i_{R} R_{L}, \\
i_{2} & =C_{0} \frac{d u_{c}}{d t}+\frac{u_{c}}{R_{L}} .
\end{aligned}
$$

Assuming $1 / \omega C_{0} \gg R_{L}$, then $i_{c} \approx 0$, at this time; it can be seen that $i_{2}=i_{R}$; thus, (1) could be shown as

$$
-M \frac{d i_{1}}{d t}=L \frac{d i_{2}}{d t}+\left(r+R_{L}\right) i_{2}
$$

When electric resistance $r+R_{L}$ is small enough or the change rate of current is large enough, there is $\omega L \gg r+R_{L}$; (3) can be changed into

$$
-M \frac{d i_{1}}{d t}=L \frac{d i_{2}}{d t} .
$$

Based on (4) it can be obtained that

$$
i_{2}=\frac{M}{L} i_{1}
$$

Substituting $L$ with $N \cdot M$ in (5), the following equation can be obtained:

$$
i_{2}=\frac{1}{N} i_{1}
$$

Therefore, the output voltage is

$$
u_{L}=i_{2} \cdot R_{L}=\frac{R_{L}}{N} i_{1}
$$

According to the above analysis, only when the sample resistance is small (typically a few ohms or less) or a large change rate of current, could formula (7) be set up. Therefore, Rogowski coil operating under the status of self-integration is suitable for measuring rapid changes in short time traveling wave pulses.

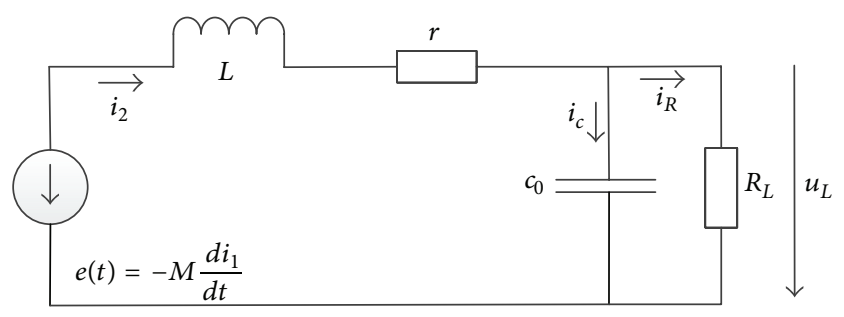

FIgURE 1: Equivalent circuit of magnetic level gauge measurement circuit.

In Figure 1, it could be known that the transfer function of Rogowski coil under the status of self-integration is as follows [19]:

$$
\begin{aligned}
H(s) & =\frac{U_{L}(s)}{I_{2}(S)} \\
& =\frac{M s}{L C_{0} s^{2}+\left(L / R_{L}+r C_{0}\right) \cdot s+\left(r / R_{L}+1\right)} .
\end{aligned}
$$

The amplitude-frequency characteristic is

$$
\begin{aligned}
& |H(j \omega)| \\
& =\frac{R_{L}}{N \cdot \sqrt{\left(1+r R_{L} C_{0} / L\right)^{2}+\left(C_{0} R_{L} \omega-\left(R_{L}+r\right) / L \omega\right)^{2}}} .
\end{aligned}
$$

The phase-frequency characteristic is

$$
\varphi(j \omega)=-\arctan \frac{C_{0} R_{L} \omega-R_{L}+r / \omega L}{1+R_{L} C_{0} / L} .
$$

Using high-frequency small-signal analysis of parallel resonant circuit theory, you can get the upper frequency and the lower frequency:

$$
\begin{aligned}
& \text { Upper frequency: } f_{H}=\frac{1}{2 \pi R_{L} C_{0}}, \\
& \text { Lower frequency: } f_{L}=\frac{R_{L}+r}{2 \pi L} .
\end{aligned}
$$

According to the above analysis of upper frequency, lower frequency, and amplitude-frequency characteristic formulas, the device based on Rogowski coil can be designed to extract fault traveling wave.

\section{PCB Traveling Wave Sensor}

3.1. Design Principle. Based on the Rogowski coil principle, PCB traveling wave sensor with simple structure, high linearity, and anti-interference ability is designed with the response frequency range of $10 \mathrm{kHz} \sim 100 \mathrm{MHz}$. Its lower frequency is $10 \mathrm{kHz}$ and the power system frequency signals and 200 times less harmonic signal can be filtered out. So PCB traveling wave sensor also has a high-pass filter function, which can be directly used in extracting high-frequency traveling wave signal in fault location and protection. 


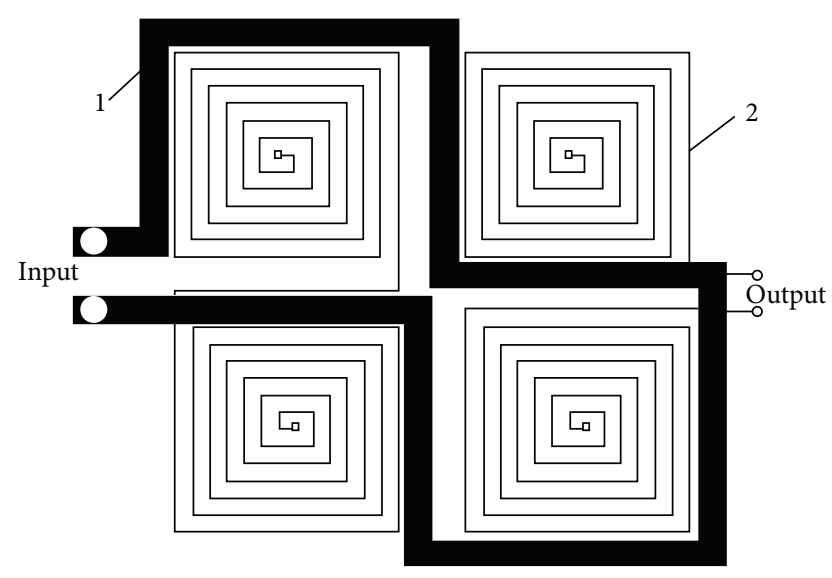

FIGURE 2: PCB traveling wave sensors schematic.

Calculation principle of PCB traveling wave sensors is derived from the law of electromagnetic induction and Ampere's law just as traditional Rogowski coil: $e(t)=$ $-M(d I / d t), M=\left(N \mu_{0} h / 2 \pi\right) \ln \left(R_{a} / R_{b}\right)\left(R_{a}\right.$ is coil diameter, $R_{b}$ is coil diameter, and $h$ is coil height).

PCB traveling wave sensor is designed by computeraided software and distributed evenly printed conductors on the printed circuit board. Printed wire replaces conventional Rogowski coil winding, and the thickness of PCB Rogowski coil replaces the traditional frame. As shown in Figure 2, 1 stands for the primary coil of the sensor and 2 stands for the secondary one. Using digital processing technology in PCB traveling wave sensor can ensure the coil equal area from the craft, winding evenly spread, and accuracy in the production. Its production can be done automatically by simply inputting the data into computer numerical control machine tools with high production efficiency and coil parameters consistency.

3.2. Design Scheme. PCB traveling wave sensor has one layer or several layers. Each layer has four subcoils or even a larger number of subcoils in the same plane (the size of each rectangular subcoil is gradually and uniformly increased, and subcoils are distributed symmetrically and sequentially connected in series). Subcoils on different layers are connected by vias. Signal output from the first and last end-sockets constitutes the secondary coil. A traveling wave sensor PCB coil tightly wound adjacent to each other on each half of the subcoils constituting one turn or loop.

The secondary coil gets a maximum induced current and the winding directions of the primary coil and the secondary coil of the subcoils are closely linked. By theoretical analysis and experimental tests, the wiring rules of primary and the secondary coils are as follows.

(1) The number of the secondary coils should be even.

(2) The adjacent secondary coils on each layer should be staggered in clockwise and counterclockwise turn.

(3) To eliminate the interference, subcoils of the secondary coils are designed to be equilateral polygon.

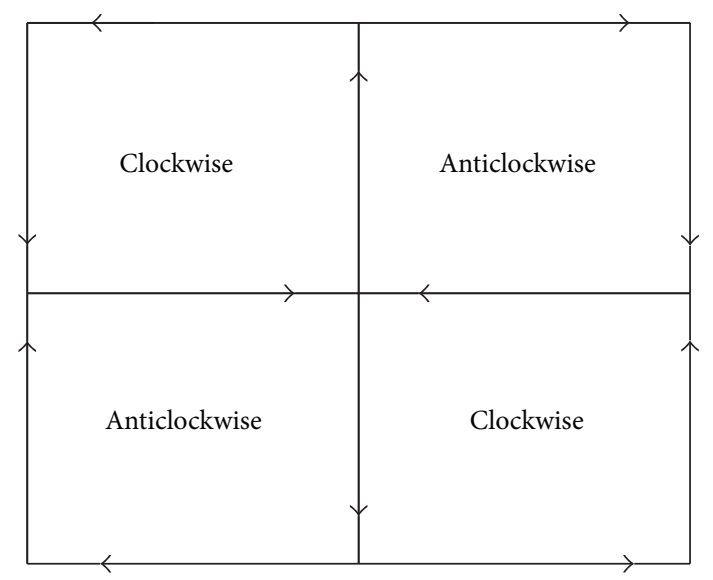

FIgURE 3: Trend of the secondary coil.

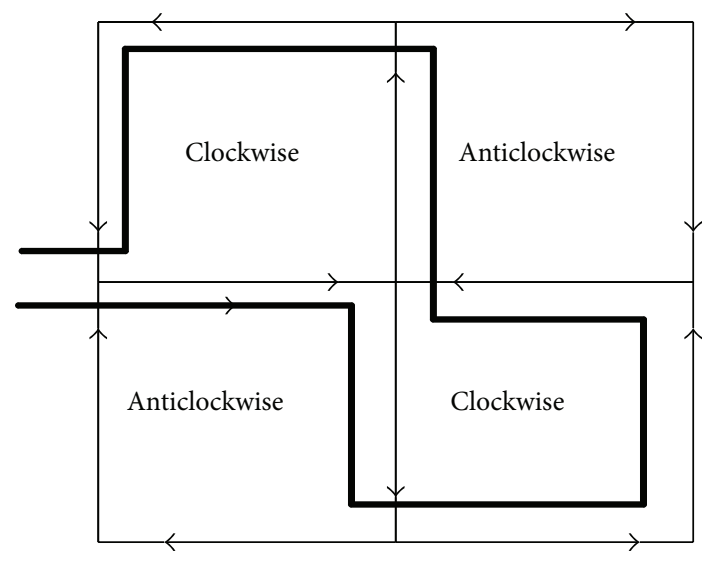

FIGURE 4: Optimal path of the primary coil.

(4) The secondary coil may be designed on one or more layers and connected in series to increase the induced current.

(5) The primary coil and the secondary coil should be matched along the optimal routing path.

As shown in Figure 3, "clockwise" and "anticlockwise" show the trend of the secondary coils. Current direction in the primary coil to make max induced current in the secondary coil is shown by the "arrow." Obviously, the optimal path of the primary coil winding is shown in Figure 4.

Following the rules above, we designed a PCB traveling wave sensor with the winding direction shown in Figure 5. It has the following characteristics.

(1) The secondary coils were distributed on two layers of the PCB.

(2) The subcoils of the secondary coils were formed hexagonal in order to reduce the reverse current in the secondary coil around the corner.

(3) The utilization rate of primary coil edges is $16 / 22$, which can induce larger current in the secondary coil. 


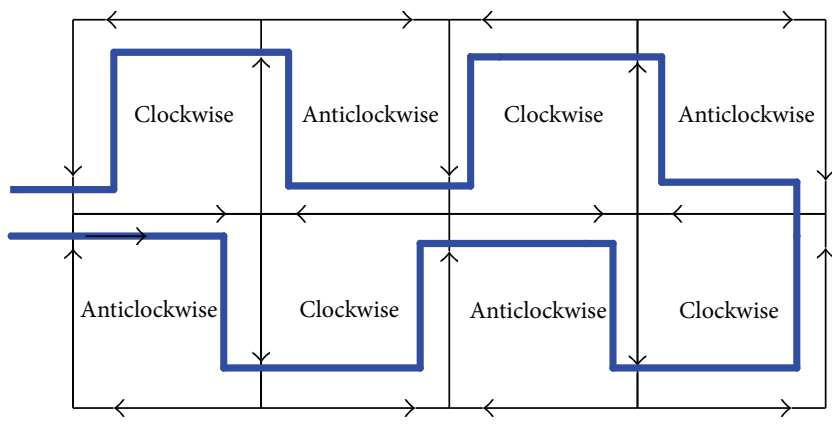

FIGURE 5: PCB traveling wave sensors winding.

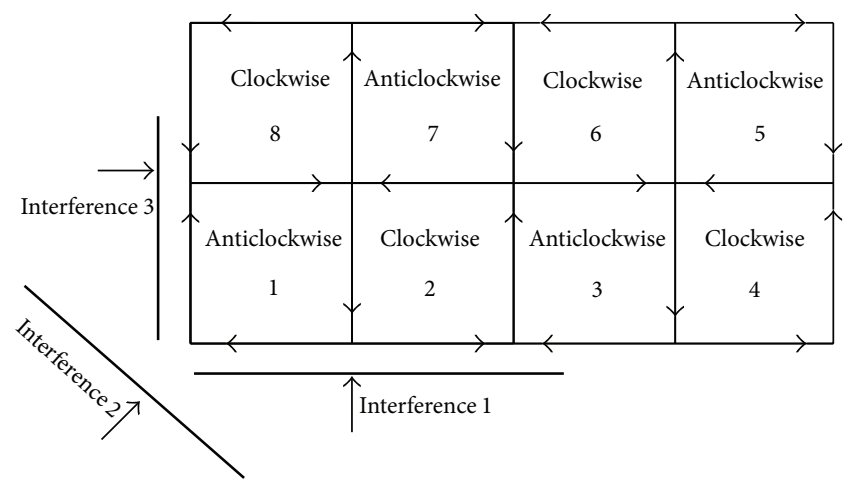

FIGURE 6: Interference analysis.

3.3. Interference Analysis. Taking the sensor winding as an example, the anti-interference analysis is shown as follows.

(1) When interference 1 is applied in a direction which is shown in Figure 6, induction coils 1 and 2, 3 and 4, 5 and 6, and 7 and 8 generate electromotive force with equal amplitude but opposite direction. The pairs of forces canceled each other out in series so that the interference cannot affect the PCB production line wave sensor.

(2) When interference 2 is applied in the direction shown in Figure 6, induction electromotive force coils 1 and 8,2 and 7, 3 and 6, and 4 and 5 produce equal and opposite direction; thus they cancel each other out in series, which does not interfere with PCB traveling wave impact sensor.

(3) When interference 3 is applied in any direction, it can be decomposed into interference 1 and interference 2 ; in this condition there is no impact on PCB traveling wave sensor. Perpendicular to the PCB interference, because of no hinge and with the secondary coil, the secondary coils will not be interfered with.

The principle of proposed PCB production line wave sensor is of the same principle with conventional Rogowski coil, which are both winding uniformly on skeleton. The difference of them is that the traditional type of wire uses enameled wire, but the planar coil based on PCB uses holes to pass through the top and bottom surface of the printed circuit board. PCB boards are all multipanel. The structure

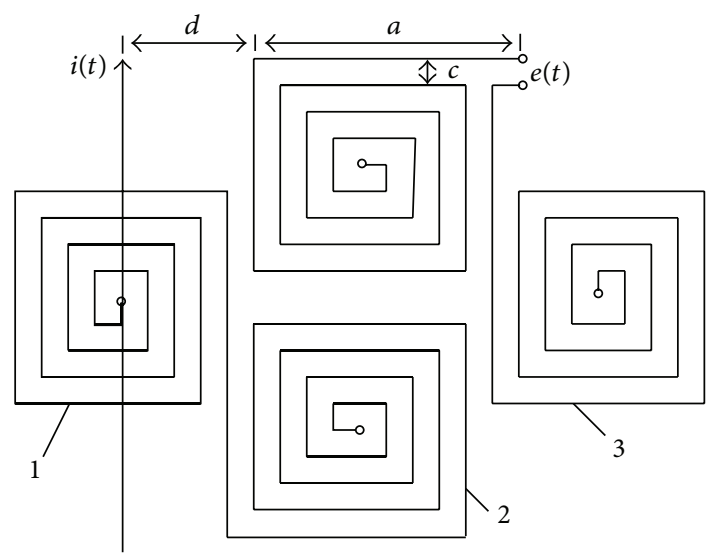

FIGURE 7: Equivalent figure of large current PCB air-core coil.

can be manufactured easily with the current designing and manufacturing process, and the winding density is symmetrical. The estimate method of self-inductance coefficient and mutual inductance coefficient is the same as the traditional Rogowski coil, but it is more with higher anti-interference ability.

\section{Mutual Inductance Calculation}

For example, in Figure 2, a width of a conductor is $D$ and has a certain thickness of the line and secondary spiral coil centerline overlap; therefore, the flux linkage effect is equivalent to the design in Figure 7.

As current $i(t)$ is flowing in the centerline of the spiral coil at position 1 , current $i(t)$ in the electromotive force induced in coil 1 is 0 . From literature [20], the current $i(t)$ on a conductor with mutual inductance coefficient of spiral coil 2 can be expressed as follows:

$$
\begin{aligned}
M= & \int_{d}^{d+a} \frac{\mu_{0}}{2 \pi x} \times a d x+\int_{d+c}^{d+a-c} \frac{\mu_{0}}{2 \pi x} \times(a-2 c) d x \\
& +\cdots+\int_{d+(n-1) c}^{d+a-(n-1) c} \frac{\mu_{0}}{2 \pi x} \times[a-2(n-1) c] d x \\
= & \frac{\mu_{0}}{2 \pi x} \sum_{k=1}^{n}[a-2(k-1) c] \frac{d+a-(k-1) c}{d+(k-1) c} .
\end{aligned}
$$

In (12), the physical significance of $a, b$, and $c$ is shown in Figure $7, n$ is the single spiral coil number, and $\mu_{0}$ is the permeability of vacuum.

So $S(d)$ and $M(d)$ can be obtained:

$$
\begin{aligned}
S(d) & =[a-2(k-1) c] \ln \frac{d+a-(k-1) c}{d+(k-1) c}, \\
M(d) & =\frac{\mu_{0}}{2 \pi} \sum_{k=1}^{n} S(d) .
\end{aligned}
$$

Figure 7 double panel (positive and negative of all four helical coils) total mutual inductance is

$$
M_{1}=2 \times[2 M(d)-M(d+a+c)] .
$$




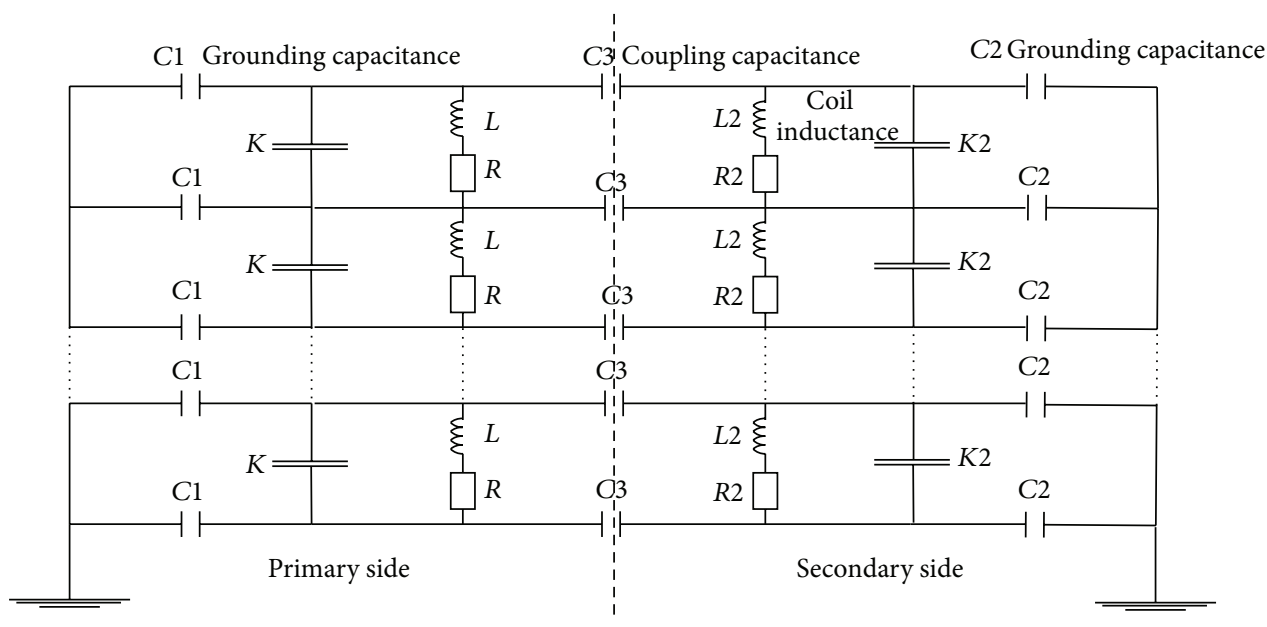

Figure 8: PCB traveling wave sensor simulation model.

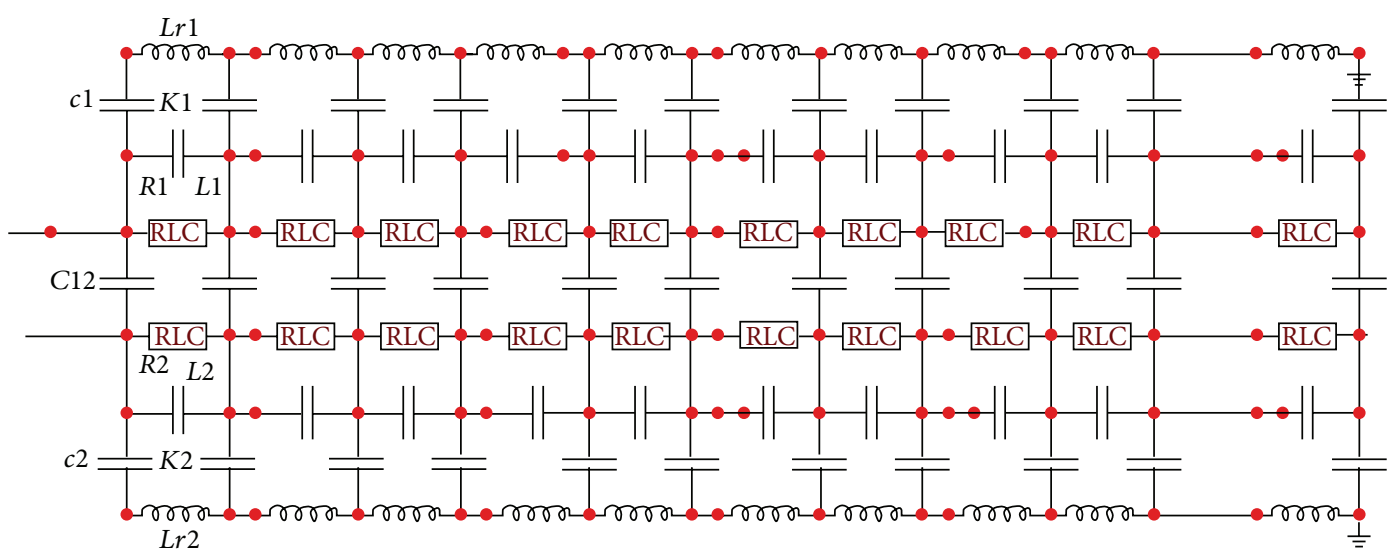

FIgURE 9: ATP simulation model of PCB traveling wave sensors.

In order to increase the coefficient of mutual inductance, the multiple double layers panel is connected in series and the induction electromotive force at the end of the combined coil is equal to each panel output accumulation of induction electromotive force:

$$
M=n M_{1}
$$

Assuming the geometries of coils of each layer are the same, their self-inductance $L$ and stray capacitance $C$ should meet the following equation:

$$
\begin{aligned}
& L=L_{1}+L_{2}+\cdots L_{n}=n L_{n}, \\
& \frac{1}{C}=\frac{1}{C_{1}}+\frac{1}{C_{2}}+\cdots+\frac{1}{C_{n}}=\frac{C_{n}}{C_{1}} .
\end{aligned}
$$

The combined natural frequency of the coil can be obtained by (16) expression:

$$
f=\frac{1}{2 \pi \sqrt{L C}}=\frac{1}{2 \pi \sqrt{n L_{1}\left(C_{1} / C_{n}\right)}}=\frac{1}{2 \pi \sqrt{L_{1} C_{1}}} .
$$

It can be seen from expression (17) that although $n$ layer PCB panel after the combination of equivalent mutual inductance coefficient increases for $n$ times, inherent frequency is constant.

\section{Simulation Analysis}

A PCB traveling wave sensor simulation model is shown in Figure 8, and the actual circuit simulation is shown in Figure 9. $R 1, L 1, R 2$, and $L 2$ are resistor and inductor per unit for high and low voltage windings (including interturn inductance and mutual inductance), respectively. Specific values of the parameters are chosen as follows: $R 1=R 2=1 \Omega, L 1=$ $29 \mathrm{mH}$, and $L 2=18 \mathrm{mH} ; C 1, C 2$ are grounding capacitance, $C 1=700 \mathrm{pF}, C 2=300 \mathrm{pF} ; K 1, K 2$ are vertical (interturn) equivalent capacitance for high and low voltage winding, respectively, $K 1=300 \mathrm{pF}, K 2=100 \mathrm{pF} ; \operatorname{Lr} 1, \operatorname{Lr} 2$ are parasitic inductance ground for high and low voltage winding, $\operatorname{Lr} 1=$ $50 \mathrm{pF}, \operatorname{Lr} 2=10 \mathrm{pF}$; $C 12$ are capacitance between windings, $C 12=22 \mathrm{pF}$. Lumped parameter $N$ is the number of units connected in cascade, and the general value of $N$ is between 10 and 20. After repeat comparison, $N$ is set to be 10 in the model. 


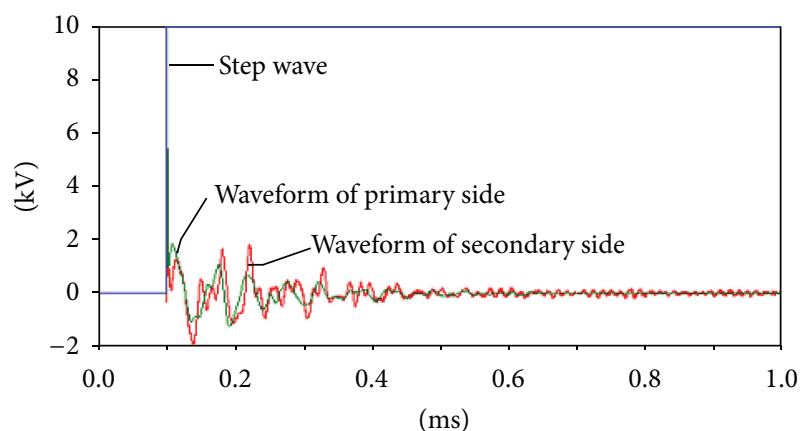

(file tr3.pl4; $x$-var $t$ ) v: XX0164-XX0012 v: XX0004-XX0158 v: XX0004

(a) When step wave simulation waveforms

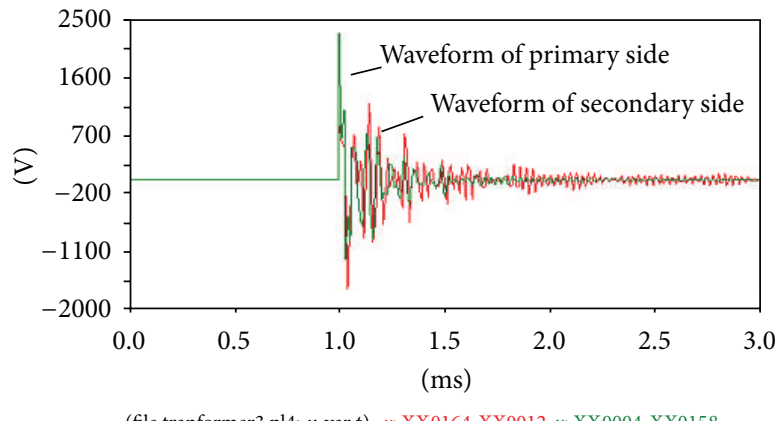

(file tranformer3.pl4; $x$-var $t$ ) $\quad v$ : XX0164-XX0012 $v$ : XX0004-XX0158

(c) When sine wave $\left(0^{\circ}\right)$ simulation waveforms

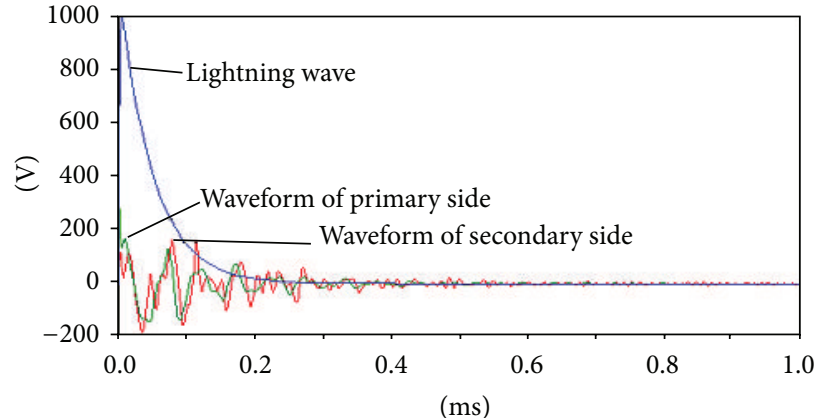

(file tranformer3.p14; $x$-var $t$ ) $v$ : XX0164-XX0012 v: XX0004-XX0158 v: XX0004

(b) When lightning wave simulation waveforms

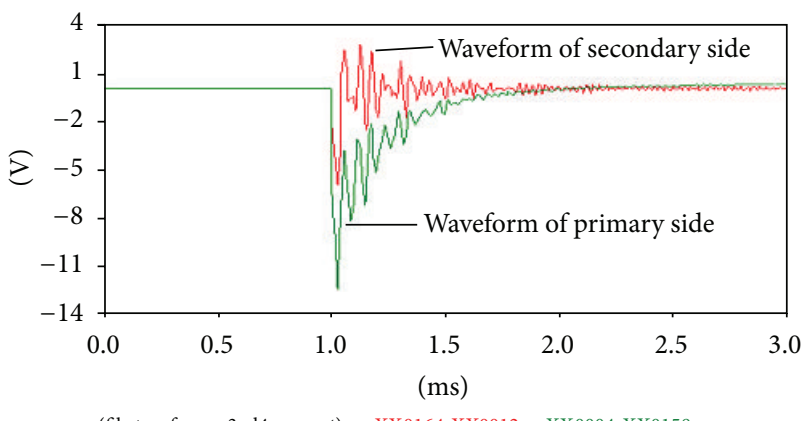

(d) When sine wave $\left(90^{\circ}\right)$ simulation waveforms

FIGURE 10: PCB traveling wave traveling wave propagation simulation waveform sensor.

Figure 9 is the EMTP simulation model. Figure 10 shows the secondary waveforms of PCB traveling wave sensor when the primary inputs are, respectively, step wave, lightning wave, and sine wave with initial phase angle are $0^{\circ}$ and $90^{\circ}$.

It could be seen from Figure 10 that the input signal and the output signal occur almost simultaneously in the inception of electrostatic induction. The electrostatic induction voltage is related to the secondary load of voltage transformer. Impact generates an oscillation signal in the second signal output circuit. When the primary coil input wave is at right angles, there is a larger secondary coil electrostatic induction voltage and a smaller electric current through the electromagnetic induction voltage. The free oscillation voltage does not exist. Secondary circuit is simulated under the right angle wave signals, the oscillation signal can be generated, and the main frequency of the oscillation is the inherent frequency of the secondary system.

\section{Conclusion}

(1) A new type of PCB traveling wave sensor has been proposed, which has large mutual inductance, strong anti-interference ability, and high measurement accuracy.

(2) Based on calculations, it has been proved that, with a simple combination, PCB traveling wave sensor is able to improve its mutual inductance effectively.
(3) Simulation results have shown that the new PCB traveling wave sensor can effectively extract and deliver traveling wave signals, thus realizing fault location and protection accurately.

\section{Conflict of Interests}

The authors declare that there is no conflict of interests regarding the publication of this paper.

\section{Acknowledgments}

The paper was supported by the National Natural Science Fund Project (51207013, 51377012), Hunan Province Science and Technology Major Project (2012FJ1003), Hunan Province Science Fund for Distinguished Young Scholars (2015JJ1001), Hunan Province Department of Education Key Projects (14A002), and Hunan Province Universities Industrialization Cultivation Project of Scientific and Technological Achievements (13CY008).

\section{References}

[1] S. Nourizadeh, M. J. Karimi, A. M. Ranjbar, and A. Shirani, "Power system stability assessment during restoration based on a wide area measurement system," IET Generation, Transmission and Distribution, vol. 6, no. 11, pp. 1171-1179, 2012. 
[2] D. R. Costianu, N. Arghira, I. Fagarasan, and S. St. Iliescu, "A survey on power system protection in smart grids," UPB Scientific Bulletin Series C: Electrical Engineering, vol. 74, no. 1, pp. 139-146, 2012.

[3] R. Mardiana, H. Al Motairy, and C. Q. Su, "Ground fault location on a transmission line using high-frequency transient voltages," IEEE Transactions on Power Delivery, vol. 26, no. 2, pp. 1298-1299, 2011.

[4] M. S. Choi, S. J. Lee, D. S. Lim et al., "A new fault location algorithm using direct circuit analysis for distribution systems," IEEE Transactions on Power Delivery, vol. 19, no. 1, pp. 35-41, 2004.

[5] H. Livani and C. Y. Evrenosoglu, "A fault classification and localization method for three-terminal circuits using machine learning," IEEE Transactions on Power Delivery, vol. 28, no. 4, pp. 2282-2290, 2013.

[6] W. Wu, Y. Lv, and B. Zhang, "On-line operating risk assessment of hidden failures in protection system," Zhongguo Dianji Gongcheng Xuebao, vol. 29, no. 7, pp. 78-83, 2009.

[7] H.-B. Jia, H.-B. Qian, and Y.-L. Qi, “Traveling-wave location for the single phase grounding fault of distribution network," Power System Protection and Control, vol. 40, no. 23, pp. 93-97, 2012.

[8] J. Qin, X. Chen, and J. Zheng, "Study on dispersion of travelling wave in transmission line," Proceedings of the CSEE, vol. 19, no. 9, pp. 27-35, 1999.

[9] M. Gilany, D. K. Ibrahim, and E. S. Tag-Eldin, "Traveling-wavebased fault-location scheme for multiend-aged underground cable system," IEEE Transactions on Power Delivery, vol. 22, no. 1, pp. 82-89, 2007.

[10] X. Zeng, Y. Zhou, Z. Liu, and G. Lin, “The sensor of travelingwave for fault location in power systems," in Proceedings of the International Conference on Power System Technology (POWERCON '04), vol. 2, pp. 1518-1521, November 2004.

[11] F. Zhang, Z.-C. Pan, H.-F. Zhang, W. Cong, and L.-L. Ma, "New algorithm based on traveling wave for location of single phase to ground fault in tree type distribution network," Proceedings of the CSEE, vol. 27, no. 28, pp. 46-52, 2007.

[12] X. Liu, K. Guo, and G. Ye, "Experimental study on the impulsevoltage transmission characteristics of inductive voltage transformers," Gaodianya Jishu, vol. 37, no. 10, pp. 2385-2390, 2011.

[13] S.-N. Luo, Z.-B. Tian, and X.-C. Zhao, "Performance analysis of air-core current transformer," Proceedings of the Chinese Society of Electrical Engineering, vol. 24, no. 3, pp. 108-113, 2004.

[14] L. Wang and B. Fang, "Simulations on transient characteristics of $500 \mathrm{kV}$ capacitor voltage transformer," Gaodianya Jishu, vol. 38, no. 9, pp. 2389-2396, 2012.

[15] T. Yamada, E. Kurosaki, N. Yamamoto, and M. Matsumoto, "Development of simple coupling-capacitor voltage transformer for GIS," in Proceedings of the IEEE Power Engineering Society Winter Meeting, pp. 269-274, February 2001.

[16] C. Xianghui, Z. Xiangjun, M. Hongjiang, L. Zewen, and D. Feng, "Rogowski sensor for power grid traveling wave based fault location," in Proceedings of the 9th International Conference on Developments in Power Systems Protection (DPSP '08), pp. 438-443, Glasgow, UK, March 2008.

[17] Q. Chen, H.-B. Li, M.-M. Zhang, and Y.-B. Liu, "Design and characteristics of two Rogowski coils based on printed circuit board," IEEE Transactions on Instrumentation and Measurement, vol. 55, no. 3, pp. 939-943, 2006.
[18] E. Abdi-Jalebi and R. McMahon, "High-performance lowcost Rogowski transducers and accompanying circuitry," IEEE Transactions on Instrumentation and Measurement, vol. 56, no. 3, pp. 753-759, 2007.

[19] X. Chu, X. Zeng, F. Deng, and L. Li, "Novel PCB sensor based on rogowski coil for transmission lines fault detection," in Proceedings of the IEEE Power and Energy Society General Meeting (PES '09), pp. 1-4, IEEE, Calgary, Canada, July 2009.

[20] K.-W. Lee, J.-N. Park, S.-H. Yang et al., "Geometrical effects in the current measurement by Rogowski sensor," in Proceedings of the International Symposium on Electrical Insulating Materials (ISEIM '01), pp. 419-422, 2001. 

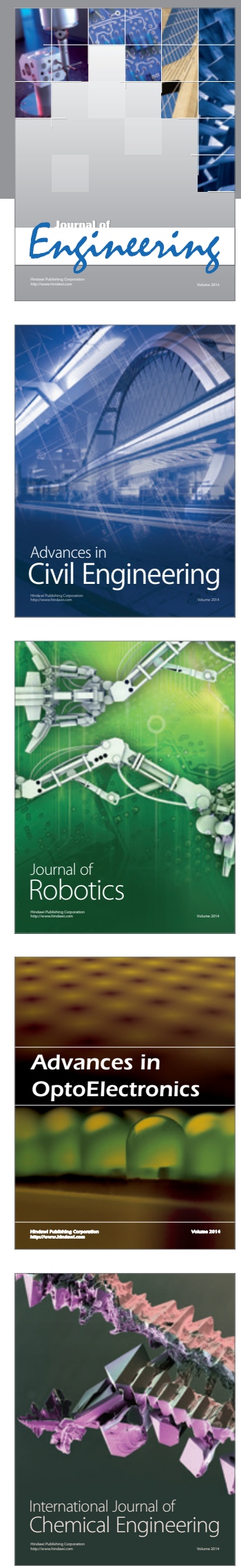

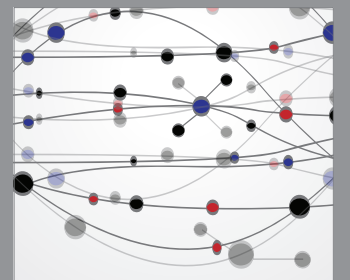

The Scientific World Journal
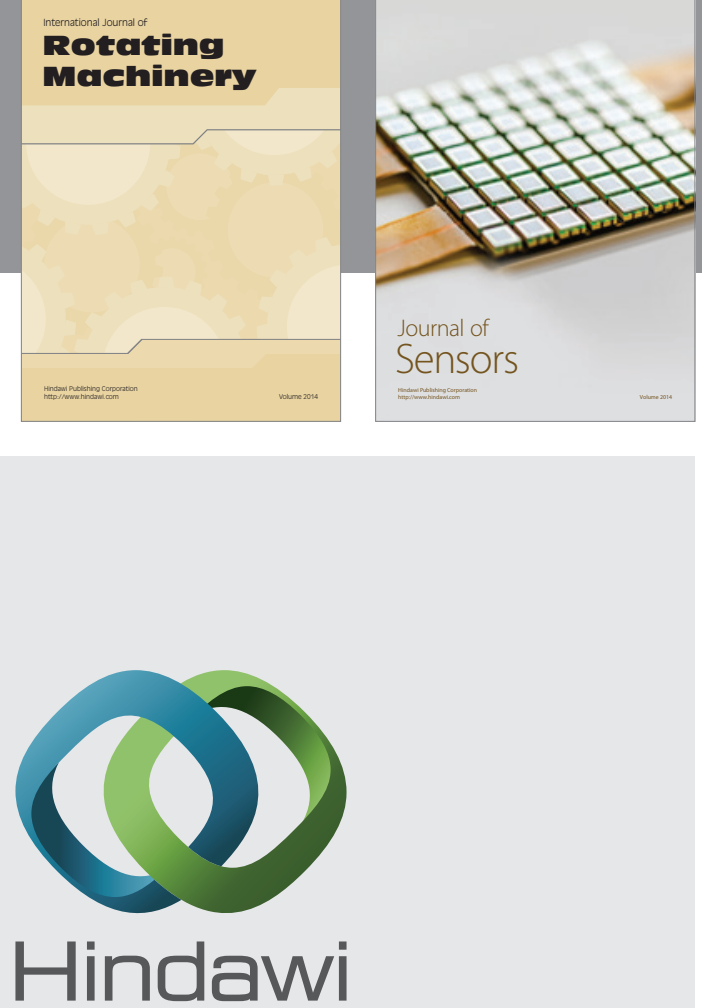

Submit your manuscripts at http://www.hindawi.com
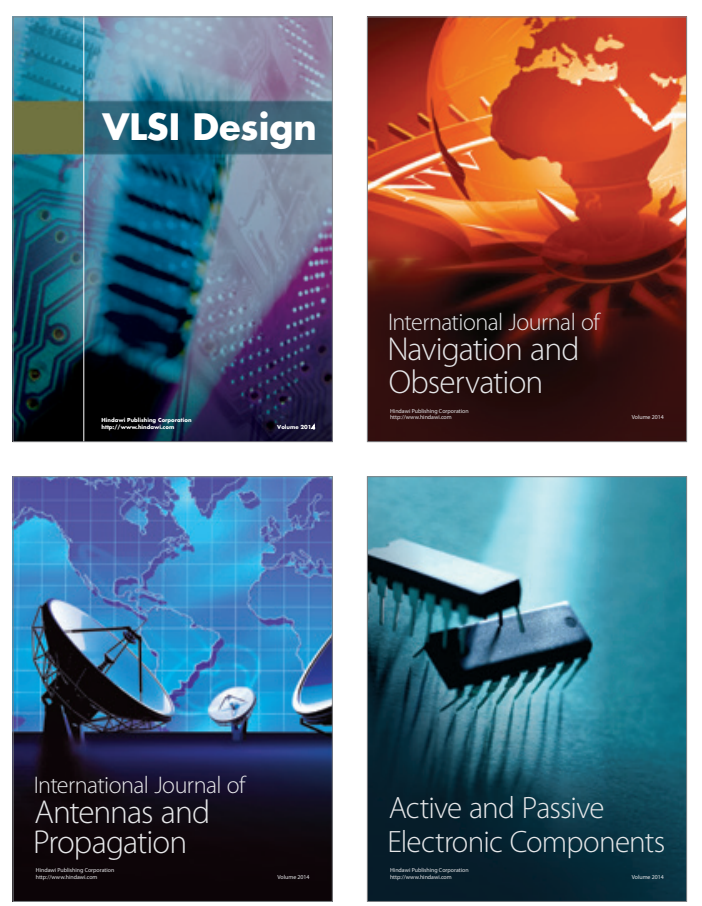
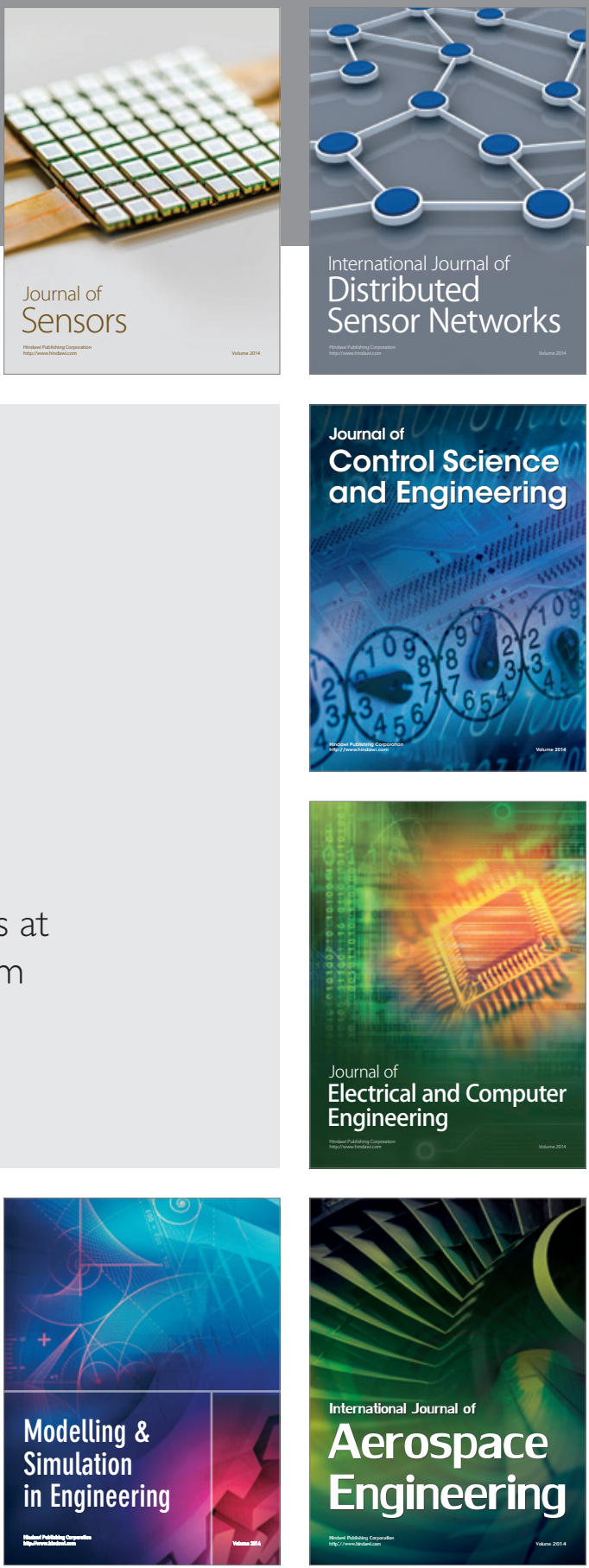

Journal of

Control Science

and Engineering
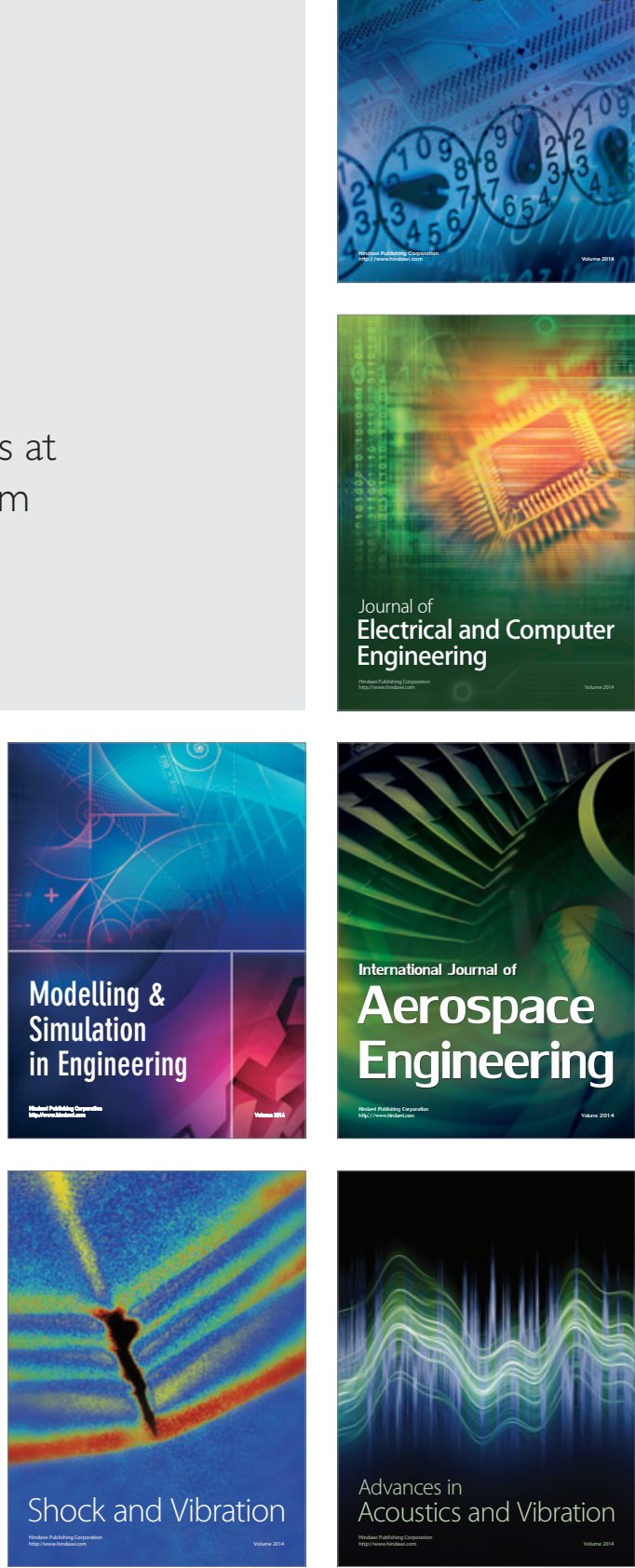